\title{
Microenvironmental contributions to hematopoietic stem cell aging
}

\author{
Ya-Hsuan $\mathrm{Ho}^{1,2}$ and Simón Méndez-Ferrer ${ }^{1,2}$ \\ ${ }^{1}$ Wellcome Trust-Medical Research Council Cambridge Stem Cell Institute and Department \\ of Haematology, University of Cambridge, Cambridge and ${ }^{2}$ National Health Service Blood \\ and Transplant, Cambridge Biomedical Campus, Cambridge, UK
}

Haematologica 2020

Volume 105(1):38-46

\section{Correspondence:}

YA-HSUAN HO

yhh29@medschl.cam.ac.uk

SIMÓN MÉNDEZ-FERRER

sm2116@cam.ac.uk

Received: July 8, 2019.

Accepted: November 14, 2019.

Pre-published: December 5, 2019.

doi:10.3324/haematol.2018.211334

Check the online version for the most updated information on this article, online supplements, and information on authorship \& disclosures: www.haematologica.org/content/105/1/38

\section{(C)2020 Ferrata Storti Foundation}

Material published in Haematologica is covered by copyright. All rights are reserved to the Ferrata Storti Foundation. Use of published material is allowed under the following terms and conditions:

https://creativecommons.org/licenses/by-nc/4.0/legalcode. Copies of published material are allowed for personal or internal use. Sharing published material for non-commercial purposes is subject to the following conditions:

https://creativecommons.org/licenses/by-nc/4.0/legalcode, sect. 3. Reproducing and sharing published material for commercial purposes is not allowed without permission in writing from the publisher.

\section{ABSTRACT}

$\mathrm{H}$ ematopoietic stem cell (HSC) aging was originally thought to be essentially an HSC-autonomous process, which is the focus of another review in the same issue of Haematologica. However, studies on the microenvironment that maintains and regulates HSC (HSC niche) over the past 20 years have suggested that microenvironmental aging contributes to declined HSC function over time. The HSC niches comprise a complex and dynamic molecular network of interactions across multiple cell types, including endothelial cells, mesenchymal stromal cells, osteoblasts, adipocytes, neuro-glial cells and mature hematopoietic cells. Upon aging, functional changes in the HSC niches, such as microenvironmental senescence, imbalanced bone marrow mesenchymal stromal cell differentiation, vascular remodeling, changes in adrenergic signaling and inflammation, coordinately and dynamically influence the fate of HSC and their downstream progeny. The end result is lymphoid deficiency and myeloid skewing. During this process, aged HSC and their derivatives remodel the niche to favor myeloid expansion. Therefore, the crosstalk between HSC and the microenvironment is indispensable for the aging of the hematopoietic system and might represent a therapeutic target in agerelated pathological disorders.

\section{Introduction to hematopoietic stem cell aging}

Adult hematopoiesis takes place in the bone marrow (BM), where hematopoietic stem cells (HSC) can self-renew, proliferate and differentiate to replenish the blood and immune systems. Given that most HSC are quiescent under homeostasis, mature blood and immune cell production is believed to derive mainly under steady state from progenitor cells (rather than HSC), which differentiate to produce mature blood cells. Cumulative studies have demonstrated that HSC are heterogeneous and contain subsets with distinct myeloid, platelet or lymphoid-biased potentials, although the existence of lymphoid-biased HSC has long been debated and remains controversial. ${ }^{1.5}$ Additionally, recent studies have shown that HSC can bypass the intermediate steps to generate mature progenies under certain conditions, such as chronic inflammation and aging.

Upon aging, HSC increase in number but their functions are impaired, characterized by reduced regenerative and homing capacity, loss of cell polarity, and myeloid-biased differentiation at the expense of lymphopoiesis. ${ }^{6.9}$ These changes were initially thought to cause only cell-intrinsic dysregulation, ${ }^{10}$ such as epigenetic deregulation, ${ }^{11}$ replication stress, ${ }^{12}$ deficient DNA repair ${ }^{13}$ and transition from canonical to non-canonical Wht signaling. ${ }^{14}$ Old HSC also suffer metabolic changes ${ }^{15,16}$ impaired autophagy ${ }^{17}$ and altered protein homeostasis, ${ }^{18}$ which contribute to the decline of their regenerative potential. However, current studies are revealing that the BM microenvironment may contribute to HSC aging. This hypothesis is supported by an elegant study in which old HSC transplanted into young recipients exhibited reduced myeloid output as compared those transplanted into old recipients, suggesting that the old BM microenvironment contributes to myeloid skewing. ${ }^{19}$ This review will cover microenvironmental contributions to 
HSC aging, provide hypotheses for BM niche remodeling based on current knowledge, and discuss the potential implications for age-related myeloid malignancies. HSCintrinsic aging mechanisms are the focus of a separate complementary review in this issue of Haematologica and will not be discussed here.

\section{Evolving views on hematopoietic stem cell niches}

HSC are surrounded by numerous cell types and the associated extracellular matrix in the BM, which form a unique microenvironment known as the "HSC niche". Osteoblasts were the first niche cells found to be involved in hematopoiesis. Early studies indicated that osteoblasts differentiate from BM osteoprogenitor cells, secrete hematopoietic cytokines and can maintain HSC in culture..$^{20}$ In 2003, two studies described for the first time that transplanted HSC localize to the bone surface of BM and their numbers are regulated by osteoblastic cells. Long-term HSC were found to adhere to spindle-shaped $\mathrm{N}$-cadherin ${ }^{+} \mathrm{CD} 45$ osteoblastic (SNO) cells, which control HSC size by BMP signaling. ${ }^{21}$ A recent study has shown that $\mathrm{N}$-cadherin ${ }^{+}$cells maintain a population of highly quiescent reserve HSC, ${ }^{22}$ suggesting the possibility that different BM niches might regulate steady-state vs. stress hematopoiesis. Another study showed that osteoblasts activated with parathyroid hormone/parathyroid hormone-related protein receptor produce high levels of Notch ligand Jagged 1 and increase HSC numbers. ${ }^{23}$ Later studies further identified Tie2/angiopoietin-1 signaling and thrombopoietin/MPL signaling as important regula- tors of HSC quiescence through interactions with osteoblasts. ${ }^{24,25} \mathrm{~A}$ high calcium concentration in the endosteum also plays an indispensable role, maintaining HSC in the endosteal niche, since calcium-sensing receptor knockout HSC fail to migrate to the endosteal BM surface after transplantation. ${ }^{26}$ In addition, the endosteal $\mathrm{BM}$ area is enriched in $\mathrm{CXCL} 12^{27}$ and stem cell factor, ${ }^{28}$ two of the most important molecules supporting hematopoiesis, strengthening the hypothesis that the endosteum is a major reservoir for HSC. However, the osteoblastic niche was thereafter challenged in studies in which osteoblasticspecific deletion of Cxcl12 or Scf only affected the maintenance of early lymphoid progenitors but had little impact on HSC. ${ }^{29}$ Furthermore, N-cadherin expression in osteolineage cells seems to be dispensable for HSC maintenance under homeostasis. ${ }^{30}$ Whereas $\mathrm{N}$-cadherin might not be essential for HSC, N-cadherin ${ }^{+}$cells appear necessary to maintain a reservoir population of quiescent HSC. ${ }^{22}$ Studies on these aspects raise the possibility that different niches might exist for activated/quiescent HSC, and/or for HSC contributing to steady-state/emergency hematopoiesis. The BM is highly vascularized, and the close developmental relationship between hematopoietic and endothelial lineages together suggest that HSC are housed and regulated in perivascular regions. To date, at least two functionally distinct perivascular niches that highly express Cxcl12 and Scf to dictate HSC cell fate have been identified in mice: (i) the arteriolar niches, composed mainly of arterioles (found throughout the BM) or endosteal transition-zone vessels, both of which are associated with sympathetic nerve fibers, Nestin-GFP bright and/or $\mathrm{NG}^{+}$cells; and (ii) the sinusoidal niches, where sinusoid-associated Cxcl12-abundant reticular cells,

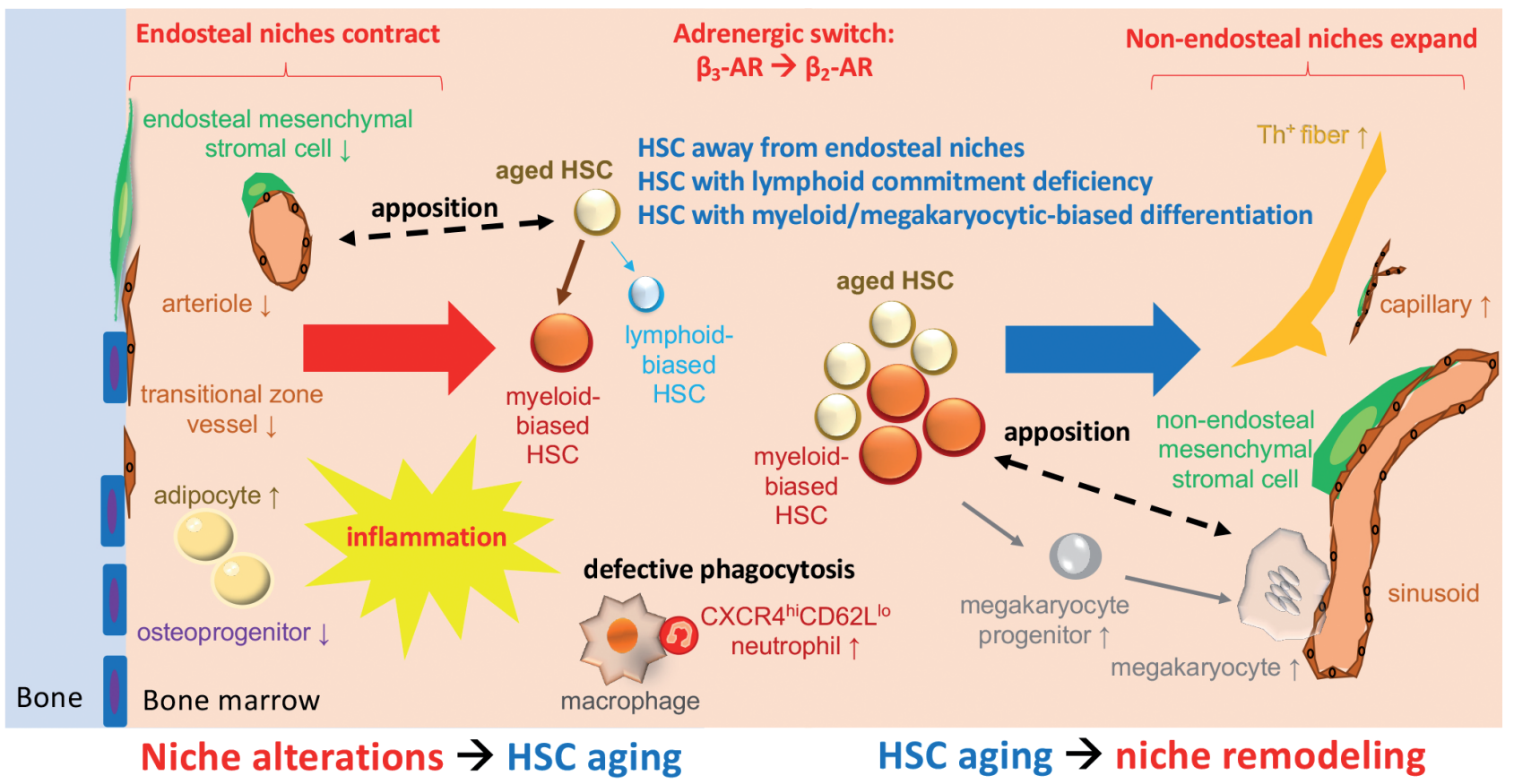

Figure 1. Schematic model of the interplay between hematopoietic stem cells and the microenvironment during aging. Loss of $\beta_{3}$-adrenergic receptor ( $\beta_{3}$-AR) activity reduces endosteal niches, pushes hematopoietic stem cells (HSC) away from the endosteum and favors myeloid bias at the expense of lymphopoiesis. Accumulation of aged HSC in the central bone marrow and increased $\beta_{2}$-AR activity causes expansion of central capillaries, myeloid cells and megakaryocytes, which locate farther from HSC. 
Nestin-GFPdim and LepR ${ }^{+}$cells are located. ${ }^{31}$ Recent studies also reveal that megakaryocytes, which are mostly adjacent to sinusoids, regulate HSC quiescence through transforming growth factor $-\beta$, thrombopoietin and platelet factor-4 secretion. ${ }^{32-34}$ Currently, it remains controversial which specialized niches predominantly regulate HSC quiescence. It is possible that HSC quiescence is differently regulated between steady-state and emergency and/or malignant hematopoiesis. However, lineage commitment appears to be influenced by the location of HSC and their derivatives in the BM. Accumulating evidence suggests that lymphopoiesis preferentially occurs near the endosteum, while myelopoiesis/erythropoiesis/megakaryopoiesis mostly takes place in non-endosteal BM regions. Supporting this concept, a recent study using $V w f-e G F P$ to label different HSC populations demonstrated that $\mathrm{Vwf}^{+}$ platelet/myeloid-biased HSC are associated with megakaryocytes, whereas Vwf lymphoid/unbiased HSC are located close to arterioles. ${ }^{35}$ Therefore, alterations in specialized niches might directly affect myeloid/lymphoid output, and the imbalanced production of mature hematopoietic cells at specific niches might in turn remodel the local microenvironment for these cells.

\section{Hematopoietic stem cells change location as niches are remodeled during aging}

A growing body of evidence has indicated that HSC redistribute within the BM upon aging. For instance, aged HSC locate away from the bone surface (endosteum), compared with young HSC, upon BM transplantation. ${ }^{36}$ This abnormal homing behavior correlates with increased BM HSC numbers and enhanced HSC egress into the circulation. ${ }^{37}$ Recent studies using whole-mount immunofluorescence staining of murine long bones further revealed that aged HSC are more distant from the endosteum, arterioles, Nestin-GFP ${ }^{\text {high }}$ cells and megakaryocytes, but HSC distance from sinusoids and Nestin-GFPlow cells appears unchanged, compared with that of young HSC. ${ }^{38-40}$ These results strongly suggest that the $\mathrm{BM}$ microenvironment is altered with age, favoring HSC lodging near nonendosteal (central) niches, over endosteal niches. The following sections will discuss current studies on age-related BM niche remodeling, the key microenvironmental players and the associated mechanisms by which HSC localization and function are regulated.

\section{Dysfunction of bone marrow mesenchymal stromal cells}

Studies regarding the absolute number of BM mesenchymal stromal cells (MSC) during aging have yielded controversial results, with some suggesting an overall increase,$^{41,42}$ while others suggest unchanged ${ }^{43,44}$ or reduced numbers. ${ }^{45}$ It is noteworthy that BM MSC are heterogeneous, and the heterogeneity in the markers used to define BM MSC immunophenotypically might explain some of these controversies. Using Nestin-gfp to label murine BM MSC, different studies have reported reduced endosteal Nestin-GFP+ ${ }^{+}$cells in the aged $\mathrm{BM}^{39,40}$ consistent with reduced numbers of arteriolar $\alpha \mathrm{SMA}^{+}, \mathrm{PDGFR} \beta^{+}$and $\mathrm{NG} 2^{+}$cells..$^{38}$ The age-related contraction of endosteal BM might initiate lymphoid deficiency, since lymphoid niches have been previously described near bone. ${ }^{2,946-48}$ However, this notion has been refined more recently after elucidating dynamic interactions between B-cell progenitors and perivascular BM MSC, which provide key signals for B lymphopoiesis (such as Cxcl12 and Il7), both in endosteal and central sinusoidal BM niches..$^{49.52}$ Functionally, old BM MSC exhibit reduced colony-forming unit-fibroblast (CFU-F) capacity in vitro and reduced expression of HSC niche factors. ${ }^{38}$ In this regard, revitalizing BM MSC to restore HSC niche factors has been proposed as a strategy to prevent DNA damage in cultured HSC. ${ }^{53}$

BM MSC exhibit reduced osteogenesis with age, which is associated with lower osteopontin secretion to the extracellular matrix. ${ }^{54}$ Osteopontin negatively regulates HSC proliferation, ${ }^{55.57}$ and its decline might accelerate HSC divisions during aging. Supporting this idea, treatment with thrombin-cleaved osteopontin partially reverses the age-associated phenotype of HSC. ${ }^{54}$

CC-chemokine ligand 5 (CCL5), a pro-inflammatory cytokine involved in bone remodeling, ${ }^{58}$ is reportedly increased with age. Researchers also reported a direct contribution to myeloid-biased differentiation at the cost of $\mathrm{T}$ cells by CCL5, ${ }^{19}$ suggesting that CCL 5 is important for aging of the hematopoietic system and the microenvironment. In contrast, old BM MSC show adipocyte skewing. ${ }^{59}$ Adipocytes are a $\mathrm{BM}$ niche component that promotes HSC regeneration after irradiation, although their roles in hematopoiesis under homeostasis seem to be dispensable ${ }^{60}$ However, altered functions of adipose tissue, including ectopic lipid deposition, insulin resistance and increased inflammation, have been described during aging. ${ }^{61}$ Accumulation of $\mathrm{BM}$ adipocytes upon aging not only reduces hematopoietic reconstitution, but also disrupts bone fracture repair. ${ }^{62}$ The latter likely contributes to the increased risk of osteoporosis and bone fracture in the elderly population. ${ }^{63,64}$

$\mathrm{BM}$ aging is also associated with senescence of $\mathrm{BM}$ MSC, evidenced by increased p53/p21-mediated DNA damage, upregulation of p16(INK4a) and elevated levels of reactive oxygen species. ${ }^{65-67}$ An age-dependent shortening of telomeres was found in telomerase-deficient $\left(\operatorname{Terc}^{-}\right) \mathrm{BM}$ MSC; consequently, lethally-irradiated Terc mice carrying wildtype BM cells display accelerated myelopoiesis. ${ }^{6}$ More recently, proteome analyses of human BM have unraveled nitric oxide synthesis and the urea cycle pathways as potential mediators for the crosstalk between old BM MSC and HSC ${ }^{69}$ Murine BM MSC show comparatively higher mRNA expression of neuronal nitric oxide synthase (encoded by the Nos1 gene), as compared with other nitric oxygen synthase isoforms, and Nos $1 \%$ mice develop certain features of premature aging, such as remodeled BM vasculature and myeloid skewing. ${ }^{39}$ Given the importance of nitric oxide in vascular biology and balanced inflammatory responses, it is likely that nitric oxide pathways participate in the aged vascular remodeling and myeloid expansion partly by modulating inflammation.

\section{Remodeling of bone marrow vasculature and endothelial cell functions}

During aging, remodeling of the BM endothelial vasculature is notable. Studies using whole-mount confocal imaging, two-photon intravital microscopy and flow cytometry analysis demonstrated overall increased vascular density in aged mice. ${ }^{38,70}$ Yet distinct vascular beds show different, or even opposite alterations with age. Arterioles appear to be decreased, while sinusoids seem 
Table 1. Microenvironmental players contributing to hematopoietic stem cell aging.

\begin{tabular}{|c|c|c|}
\hline Players & Size & Functions/Mechanisms \\
\hline Mesenchymal lineages & $\begin{array}{l}\text { Decreased endosteal Nestin-GFP+ }{ }^{+} \text {cells } \\
\text { Increased non-endosteal Nestin-GFP+ cells } \\
\text { Decreased } \alpha \mathrm{SMA}^{+} \text {cells, } \mathrm{NG2}^{+} \text {cells, PDGF } \beta^{+} \text {cells }\end{array}$ & $\begin{array}{l}\text { Increased adipogenesis } \\
\text { Decreased osteogenic differentiation } \\
\text { Cellular senescence } \\
\text { Altered nitric oxide, urea cycle pathways } \\
\text { Reduced secretion of niche factors } \\
\text { HSC closer to non-endosteal niches, away from endosteal niches }\end{array}$ \\
\hline Endothelial cells & $\begin{array}{l}\text { Increased overall vascular density } \\
\text { Decreased arterioles; shortened arteriole segments } \\
\text { Unchanged/preserved sinusoids } \\
\text { Decreased transitional zone vessels } \\
\text { Increased small capillaries }\end{array}$ & $\begin{array}{l}\text { Increased vascular leakiness } \\
\text { Vasodilation } \\
\text { Decreased angiogenic potential } \\
\text { Decreased Notch activity } \\
\text { HSC away from arterioles } \\
\text { HSC preserved in sinusoids } \\
\text { DLL4 regulating HSC myeloid skewing } \\
\text { Sinusoidal Jag2 regulating HSC proliferation }\end{array}$ \\
\hline Inflammatory cytokines & Increased IL-1, IL-3, IL-6, TNF $\alpha$, INF $\gamma$, TGF $\beta$ & $\begin{array}{l}\text { IL-1 } \beta \text { regulating HSC myeloid-skewing } \\
\text { IL- } 6 \text { regulating megakaryocyte differentiation } \\
\text { TNF } \alpha \text { protecting HSC from necroptosis } \\
\text { TGF- } \beta \text { regulating megakaryopoiesis } \\
\text { IFN regulating megakaryocytic bias }\end{array}$ \\
\hline $\begin{array}{l}\text { Sympathetic nervous } \\
\text { system }\end{array}$ & Increased $\mathrm{Th}^{+}$nerve fibers & $\begin{array}{l}\beta_{2}-\text { AR activation regulating HSC myeloid-skewing toward platelet production } \\
\beta_{3} \text {-AR inactivation regulating niche remodeling, HSC lymphoid deficiency } \\
\text { Functional switch of } \beta \text { adrenergic signaling ( } \beta_{2} \text {-AR overriding } \beta_{3} \text {-AR) }\end{array}$ \\
\hline Others & $\begin{array}{l}\text { Increased megakaryocytes } \\
\text { Accumulation of CXCR } 4^{\text {igh }} C D 62 L^{\text {low }} \text { senescent } \\
\text { neutrophils }\end{array}$ & $\begin{array}{l}\text { Megakaryocytes closer to sinusoids } \\
\text { HSC away from megakaryocytes } \\
\text { Macrophages with impaired phagocytosis }\end{array}$ \\
\hline
\end{tabular}

GFP: green fluorescent protein; $\alpha$ SMA: alpha smooth muscle actin; PDGF $\beta$ : platelet-derived growth factor; HSC: hematopoietic stem cells; IL: interleukin; TNF $\alpha$ : tumor necrosis factor alpha; INF $\gamma$ : interferon gamma; TGF $\beta$ : transforming growth factor beta; DLL4: delta-like 4; Jag2: Jagged 2; AR: adrenergic receptor.

unchanged upon aging. ${ }^{39}$ Consistent with these observations, arteriole segments covered by Nestin-GFPright cells appear shortened. ${ }^{8}$ Transitional zone vessels containing type-H endomucin (EMNC)-high endothelial cells (which are enriched in the murine trabecular BM, where they support developmental bone growth ${ }^{71}$ ) are reduced in old mice. ${ }^{39,70}$ In contrast, small capillaries (CD31 high EMCN- cells $<6 \mu \mathrm{m}$ in diameter) are notoriously expanded in the central marrow. ${ }^{39}$

The functionality of vascular endothelium declines with age, as manifested by increased vascular leakiness, increased levels of reactive oxygen species and decreased angiogenic potential. ${ }^{72}$ Poulos et al. previously reported that HSC purified from young mice and co-cultured with endothelial cells from old mice lack long-term hematopoietic multilineage reconstitution, while old HSC co-cultured with young endothelial cells maintain their selfrenewal ability. ${ }^{72}$ Infusion of young endothelial cells into aged, conditioned mice revives the old hematopoietic system. Kusumbe et al. identified high Notch activity in type$\mathrm{H}$ endothelial cells and their associated subendothelial/perivascular cells, ${ }^{70}$ suggesting that contraction of endosteal vessels upon aging concomitantly occurs with impaired Notch signaling. Overexpression of the Notch ligand D114 in vascular endothelial cells can prevent myeloid skewing of hematopoietic progenitors ${ }^{73}$ but cannot completely rescue HSC aging, ${ }^{70}$ perhaps consistent with the finding of another study in which Dll4 was unchanged in the aged murine $\mathrm{BM}^{40} \mathrm{~A}$ common finding is reduced endosteal activity of Notch ligand, since the latter study reported reduced expression of Jagged2 (Jag2) ligand in aged Nestin-GFPhigh cells. In contrast, Jag2 levels seem increased in the sinusoids, or their associated NestinGFPlow cells. Moreover, Jag2 blockade induces proliferation and clustering of aged HSC near the sinusoids. Therefore, whereas the specific role of Dll4 during aging is not clear, alterations of Notch signaling do seem to be important for hematopoietic aging. Together, these results strongly suggest that altered Notch signaling critically contributes to HSC aging in different ways depending on the niche: in the endosteal vessels, Notch signaling appears to regulate HSC lineage commitment, whereas it is required in the sinusoids to preserve old HSC (since HSC accumulate in sinusoidal niches as a function of age). ${ }^{40}$

\section{Inflammation}

Aging of the BM microenvironment is associated with increased pro-inflammatory cytokines, both in mice and humans. ${ }^{74}$ Several lines of evidence have indicated that these inflammatory cytokines drive myeloid/megakaryocytic differentiation. In aged-related myeloid malignancies, such as myeloid proliferative neoplasms and chronic myelogenous leukemia, serum interleukine (IL)- $1 \beta$ and IL6 levels are elevated. ${ }^{75,76}$ Pietras et al. reported that chronic IL-1 exposure induces HSC myeloid skewing at the expense of self-renewal. ${ }^{77}$ IL- $1 \alpha / \beta$ regulates thrombopoiesis in vitro, ${ }^{78,79}$ possibly explaining high platelet counts in aged mice. ${ }^{80}$ Defective phagocytosis of macrophages during aging induces expansion of plateletbiased HSC through Il-1 $\beta$ signaling. ${ }^{81}$ Il- 6 promotes thrombopoiesis either through a direct effect on BM megakaryocyte differentiation $^{39}$ or indirectly upregulating hepatic 
thrombopoietin levels. ${ }^{82}$ Yamashita et al. demonstrated that transient stimulation of tumor necrosis factor- $\alpha$ prevents HSC from necroptosis, and proposed that constitutive activation might lead to hyperproliferation of HSC and exacerbated myelopoiesis in aging and myeloproliferative disorders. ${ }^{83}$ Megakaryocytes express transforming growth factor- $\beta$ to regulate HSC quiescence, while megakaryocyte-derived transforming growth factor- $\beta$ also stimulates thrombopoietin synthesis by BM stromal cells to enhance megakaryopoiesis. ${ }^{84}$ An elegant study by Haas et al. found that acute inflammation induces proliferation of the stem cell-like megakaryocyte progenitor to quickly replenish platelet loss, and the process is in part mediated through the interferon family. ${ }^{85}$

Despite the well-known lymphocyte deficiency associated with aging, only the frequency and function (but not the absolute number of lymphoid-biased/balanced HSC) appear to decline with age..$^{66}$ In fact, during aging both platelet/myeloid-biased and lymphoid-biased HSC expand, but exhibit altered gene expression programs and myeloid and platelet-skewing. ${ }^{80}$ These findings suggest a cell fate change of HSC upon aging, and the net outcome is an increase of the myeloid/platelet compartment at the expense of the lymphoid compartment. Two possible non-mutually exclusive explanations are: (i) different HSC suffer the same intrinsic abnormalities upon aging and (ii) microenvironmental alterations specifically influence HSC and direct their cell fate. In support of the second possibility, distinct HSC subpopulations respond differently to inflammatory challenges during aging. ${ }^{87}$ Moreover, old lymphoid-biased/balanced HSC seem to retain normal lymphoid commitment potential when removed from an old microenvironment. ${ }^{5}$ Exogenous addition of IL-1 can block lymphocyte differentiation from old lymphoidbiased HSC, confirming the indispensable role of IL-1 in HSC fate decisions. Consistently with this notion, IL-1 blockade seems sufficient to revert the age-dependent increase of megakaryocytic-biased HSC in vitro. ${ }^{81}$ IL-1 blockade can also attenuate myeloid expansion and inflammatory arthritis associated with the elderly. ${ }^{88}$

However, whether the inflamed BM niche is the cause or the consequence of HSC aging remains debated. It is notable that mature myeloid/megakaryocytic cells are a major source of inflammatory cytokines. ${ }^{89}$ Therefore, exacerbated myelopoiesis during aging might induce myeloid/megakaryocytic HSC skewing through inflammatory remodeling of the BM microenvironment. Many different cytokines directly affecting HSC (e.g., IL-6, tumor necrosis factor and interferon) increase during aging. A positive feedback loop between the myeloid cells and their derived inflammatory cytokines might increase both myelopoiesis and the cytokine storm. Future studies are needed to clarify the roles of other inflammatory cytokines, such as IL-6, in the regulation of HSC during aging.

\section{Neuronal regulation by sympathetic adrenergic signaling}

It has been reported that $\mathrm{BM}$ sympathetic stimulation of $\beta_{2}$ or $\beta_{3}$ adrenergic receptors (AR) regulates the egress and granulocyte - colony-stimulating factor -induced mobilization of HSC. ${ }^{90-92}$ A recent publication has suggested that noradrenergic nerve fibers are reduced in old murine
$\mathrm{BM} .^{38}$ The study also indicated that surgical denervation of young $\mathrm{BM}$ induces premature aging of the hematopoietic system, although the inflammation caused by the surgical denervation might have had a certain influence. A similar reduction of nerve fibers was found in a mouse model of an age-related blood disorder, myeloproliferative neoplasm, ${ }^{75}$ suggesting that BM neuropathy might predispose to myeloid malignancies with age. However, a recent study did not find such a reduction of BM noradrenergic fibers in aged mice. ${ }^{93}$ Moreover, whole-mount imaging and three-dimensional reconstruction of different bones has revealed doubled BM area covered by noradrenergic nerve fibers in aged mice. ${ }^{39}$ This result is consistent with the well-known increase in sympathetic activity in the elderly, manifested for instance by an increased concentration of noradrenaline in the human plasma with age. ${ }^{94-97}$ Increased sympathetic activity causes myeloid skewing of old HSC through activating $\beta_{2}-A R$, since exacerbated thrombopoiesis is present in old wildtype mice but absent in old $A d r b 2^{-\alpha}$ mice or Adrb2 $2^{-\alpha} A d r b 3^{--}$mice. ${ }^{39}$ A previous study showed that $\alpha$-AR directly regulated megakaryocyte migration, adhesion and proplatelet formation under stress, but $\alpha$-AR did not affect the earlier commitment of progenitor cells to the megakaryocyte lineage. ${ }^{98}$ Therefore, the age-dependent increase in sympathetic innervation might activate different AR as hematopoietic progenitor cells differentiate along the megakaryocyte lineage. Additionally, the sympathetic nervous system is known to control inflammation in a context-dependent manner. ${ }^{99}$ The concentration of catecholamines and the levels of expression of different AR influence the inflammatory state of innate immune cells. Increased sympathetic activity during aging might contribute to the cytokine storm by activating inflammatory cells, and subsequently affect lineage-bias of HSC.

Interestingly, $\beta_{3}$-AR exhibits opposite effects on lymphomyeloid skewing, compared with $\beta_{2}$-AR. Adult Adrb3 mice show a decreased frequency of endosteal lymphoidbiased HSC and/or lymphoid multipotent progenitors. ${ }^{38,39}$ Age-related remodeling of vasculature, such as reduced transitional zone vessels and expanded small capillaries, possibly explains the lymphoid deficiency in these mice. ${ }^{39}$ Altogether, these results suggest that lack of $\beta_{3}$-AR in the microenvironment might accelerate aging of the hematopoietic system. One study suggested that administration of a $\beta_{3}$-AR agonist to old mice rejuvenates most features of HSC aging, but overall changes of hematopoiesis in peripheral blood were not reported in this study. ${ }^{38}$ The same $\beta_{3}-A R$ agonist reduced myeloid expansion in a mouse model of myeloproliferative neoplasm ${ }^{75}$ and a murine model of Hutchinson-Gilford progeria syndrome (HGPS). ${ }^{39}$ However, hematopoietic rejuvenation was not detected in the peripheral blood of mice treated with this $\beta_{3}$-AR agonist over 40 weeks in another study. ${ }^{75}$ Likewise, elderly individuals with myeloproliferative neoplasms who received a $\beta_{3}$-AR agonist over 24 weeks did not show rejuvenation in the peripheral blood counts in a human study. ${ }^{100}$ Five-month old mice lacking $\beta_{3}$-AR reportedly showed normal peripheral blood counts ${ }^{39}$ or premature, lymphoid deficiency and myeloid skewing. ${ }^{38}$ The discrepancies between these studies suggest that modulating a single neuronal pathway might not be sufficient to rejuvenate overall hematopoiesis. This may be due to the multiple compensatory/adjustment mechanisms of the autonomic nervous system revealed, for instance, in the cardio- 
vascular system. ${ }^{101}$ It is worth mentioning that myeloid and lymphoid cells are also a source of catecholamines. ${ }^{102}$ Adrenal gland-derived adrenaline and immune cell-derived (nor)adrenaline might contribute to increased levels of adrenaline and noradrenaline in the circulation of aged individuals ${ }^{94.97}$ which, together with the increased BM noradrenergic innervation, might activate BM AR. We propose that sympathetic regulation of lympho-myeloid skewing pivots on activation or inactivation of different AR. A functional switch of neurotransmission $\left(\beta_{2}\right.$-AR overriding $\beta_{3}-A R$ ) with age, rather than a general decline of $B M$ noradrenergic innervation, ${ }^{38}$ might initiate $\mathrm{BM}$ niche remodeling and subsequently promote HSC myeloid skewing toward platelet production. Further investigation of other adrenergic and/or cholinergic signaling pathways possibly influencing aging is warranted.

\section{Other players in the bone marrow microenvironment}

Emerging data suggest that the progeny of HSC can feed back to regulate their activity under homeostasis, raising the possibility that mature hematopoietic cells (or their interactions with others) also contribute to HSC aging. For instance, clearance of senescent CD62L $\mathrm{L}^{\text {low }}$ CXCR $4{ }^{\text {high }}$ neutrophils by macrophages has been reported to modulate HSC niches. ${ }^{103}$ Frisch et al. discovered that aged macrophages are unable to engulf senescent neutrophils, leading to expansion of megakaryocytic-biased HSC through IL-1 $\beta$ signaling. ${ }^{81}$ Another key player is the megakaryocyte, reportedly expressing CXCL4, thrombopoietin and transforming growth factor- $\beta$ to control HSC proliferation/quiescence. ${ }^{32-34}$ In murine BM, around $20 \%$ of HSC are spatially associated with megakaryocytes, ${ }^{32}$ and depletion of megakaryocytes expands plateletbiased HSC. ${ }^{35}$ During aging, megakaryocytes are found in increased numbers, lodging closer to sinusoids, and abundantly forming pseudopodial extensions (likely to be proplatelets). ${ }^{39}$ Of note, the distance between HSC and megakaryocytes increases substantially, ${ }^{38,39}$ suggesting a remodeling of megakaryocyte niches with age. It is possible that megakaryocytes with these morphological changes fail to anchor HSC, inducing HSC hyperproliferation and lineage bias. However, whether and how agerelated alterations of megakaryocytes regulate HSC aging is still unknown and requires further investigation.

\section{Premature aging in Hutchinson-Gilford progeria syndrome}

In HGPS, aberrant splicing of the LMNA gene (encoding lamin A and C) leads to nuclear assembly of the truncated protein, prelamin A (progerin). ${ }^{104,105}$ Certain hallmarks of murine hematopoietic aging, such as increased platelet counts, have been observed in HGPS. ${ }^{106}$ Given that cells aging naturally also express increased levels of progerin, ${ }^{107}$ it is possible that normal physiological conditions and progeria might share some aging mechanisms. Grigoryan et al. recently reported that HSC deficient of LMNA display a premature aging-like phenotype, ${ }^{108}$ suggesting a prominent role of lamin $\mathrm{A} / \mathrm{C}$ in hematopoiesis at the level of HSC. The strong impact of progeria on growth and sexual maturation might be paralleled by altered endocrine regulation of HSC, since growth hormones and sex hormones regulate HSC survival, proliferation and lineage commitment. ${ }^{109-112}$ However, it remains unknown whether premature hematopoietic aging in HGPS is a consequence of progerin accumulation in HSC, other hematopoietic cells and/or the microenvironment. Using the Lmna ${ }^{66096 / 6609 G}$ mouse model, ${ }^{113}$ we found that primary

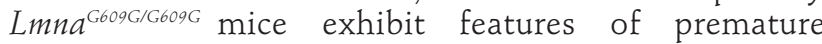
hematopoietic aging; however, premature hematopoietic aging is not observed in wildtype recipients carrying Lmna ${ }^{6609 G / 6609 G}$ hematopoietic cells. ${ }^{39}$ Microenvironmental alterations are observed in Lmna ${ }^{66096 / 6609 G}$ mice, some of which are shared between normally aged mice, such as elevated levels of pro-inflammatory cytokines (IL-3, IL-6, IL-1, interferon- $\gamma$ ), increased megakaryocytes with proplatelet-like structure and megakaryocyte apposition to $\mathrm{BM}$ sinusoids. Of note, $\beta_{3}$-AR agonism improves exacerbated myeloid expansion and restores the apposition of HSC to megakaryocytes. These results suggest that premature hematopoietic aging in HGPS is not HSCautonomous, and certain aging features can be rejuvenated by targeting the microenvironment. ${ }^{39}$

\section{Aged hematopoietic stem cells/ microenvironment: chicken or egg?}

One remaining key question relates to whether microenvironmental alterations initiate HSC aging and/or whether old HSC cause niche remodeling. It is noteworthy that HSC aging is not characterized by a single cellular feature, and different aging features might emerge individually at different developmental stages. For instance, in murine aging, defective lymphopoiesis starts early, at the age of 8 months old, ${ }^{114}$ while increased platelet counts do not seem to be pronounced until 18 months. ${ }^{39} \mathrm{BM}$ noradrenergic nerve fibers appear to be decreased in adult, 8month old mice ${ }^{75}$ (Supplementary Figure $5 \mathrm{~B}$ in the report of that study), but these fibers appear increased in aged (20-month old) mice, when $\beta_{3}-A R$ signaling is already strongly reduced. ${ }^{39} \mathrm{~A}$ deficiency in $\beta_{3}$-AR accelerates the loss of endosteal lymphoid-biased HSC in 4-month old mice, but it does not aggravate HSC aging in old mice. ${ }^{39}$ In contrast, a deficiency in $\beta_{2}$-AR impairs megakaryopoiesis in young and old mice, and double knockout mice for $\beta_{2}$ $\mathrm{AR}$ and $\beta_{3}$-AR recapitulate the hematopoietic phenotype of single $\beta_{2}$-AR-deficient mice. These results suggest that $\beta_{2}$-AR overrides $\beta_{3}$-AR during aging, and that this adrenergic remodeling contributes to imbalanced lymphoid/myeloid output. We propose that a lack of $\beta_{3^{-}}$ AR activity near endosteum initiates the contraction of endosteal niches, which attenuates lymphopoiesis, favors myeloid bias and pushes HSC away from the endosteum. An adrenergic switch from $\beta_{3}$-AR to $\beta_{2}$-AR could feed back to worsen the reduction of endosteal niches, since activation of $\beta_{2}-A R$ on osteoblasts is known to restrain bone formation. ${ }^{115}$ However, the cell types expressing $\beta_{2}$-AR and/or $\beta_{3}$-AR involved in aged hematopoiesis are still elusive. Chances are that cells highly expressing $\beta_{2}$-AR replace those with high expression of $\beta_{3}$-AR over time, or BM niche cells ubiquitously increase $\beta_{2}$-AR while decreasing $\beta_{3}$-AR upon aging. These and other hypotheses need to be validated in future studies ${ }^{116}$ since the experiments were performed using global knockouts. Tissue-specific deletion of $\beta_{2}$-AR and/or $\beta_{3}$-AR will provide further insights into the mechanisms of $\beta$-adrenergic switching during aging. Importantly, $\beta_{2}$-AR and $\beta_{3}$-AR have different affinities for noradrenaline and adrenaline; whereas $\beta_{3}$-AR 
shows higher affinity for noradrenaline over adrenaline, the opposite is true for $\beta_{2}$-AR. ${ }^{117}$ Another possibility might be that BM concentrations of both neurotransmitters differ in aging, leading to imbalanced stimulation of the two receptors. The observation that old HSC home in the BM away from endosteal regions suggests that HSC-driven niche remodeling mainly occurs in non-endosteal domains. For instance, skewed myelopoiesis leading to increased numbers of neutrophils and defective phagocytosis of marrow macrophages might modulate the microenvironment favoring myeloid bias during aging. ${ }^{81}$ Increased myeloid cells might provide an additional source of catecholamines in a feed-forward loop promoting megakaryocyte differentiation by activating $\beta_{2}$-AR. We propose that accumulation of old HSC results in microenvironmental remodeling by reinforcing adrenergic activity, expansion of non-endosteal niches and enhanced myeloid/megakaryocyte differentiation. As a secondary outcome, alteration of megakaryocyte niches (increased megakaryocyte numbers, proplatelet formation and apposition to sinusoids) might release HSC from their state of quiescence, further promoting HSC proliferation with age.

\section{Niche alterations might predispose to hematologic neoplasms}

The risk of developing myeloid malignancies increases significantly in individuals harboring clonal hematopoiesis-related somatic mutations. In fact some of these mutations are oncogenic drivers of myeloid malignancies. ${ }^{118}$ However, the factors limiting clonal expansion or, instead, allowing the mutant clones to become dominant and, in some cases, cause disease, remain unclear. Interestingly, similar niche alterations are shared between aging and myeloid disorders associated with age, which is an important selection pressure to expand aberrant HSC clones. For instance, a damaged neuro-MSC circuit promotes the development of a cytokine storm created by the mutant HSC, aggravating the progression of myeloproliferative neoplasms. ${ }^{75}$ In myelodysplastic syndromes, abnormal production of cytokines from the microenvironment, dysfunction of MSC and osteolineage cells, and vascular remodeling have been associated with disease initiation and progression. ${ }^{119,20}$ Targeting the abnormal microenvironment could be a promising complementary therapeutic approach to treat hematologic cancers in the future, especially when early diagnosis becomes available.

\section{Conclusive remarks}

Aging of the hematopoietic system might result from both HSC-intrinsic and microenvironmental alterations with changes in the location, function and regulation of HSC and their progeny. Future studies will determine the relative contribution of the aged microenviroment to altered hematopoiesis and increased incidence of agerelated disorders originating in the $\mathrm{BM}$.

\section{Acknowledgments}

Y-HH received fellowships from Alborada Scholarship (University of Cambridge), Trinity-Henry Barlow Scholarship (University of Cambridge) and R.O.C. Government Scholarship to Study Abroad (GSSA). This work was supported by core support grants from the Wellcome Trust and the MRC to the Cambridge Stem Cell Institute, National Health Service Blood and Transplant (United Kingdom), European Union's Horizon 2020 research (ERC-2014-CoG-64765) and a Programme Foundation Award from Cancer Research UK to SM-F The authors regret that some relevant literature could not be discussed because of space constrictions.

\section{References}

1. Carrelha J, Meng Y, Kettyle LM, et al. Hierarchically related lineage-restricted fates of multipotent haematopoietic stem cells. Nature. 2018;554(7690):106-111.

2. Weksberg DC, Chambers SM, Boles NC, Goodell MA. CD150- side population cells represent a functionally distinct population of long-term hematopoietic stem cells. Blood. 2008;111(4):2444-2451.

3. Kiel MJ, Yilmaz OH, Morrison SJ. CD150cells are transiently reconstituting multipotent progenitors with little or no stem cell activity. Blood. 2008;111(8):4413-4414; author reply 4414-4415.

4. Kent DG, Copley MR, Benz C, et al. Prospective isolation and molecular characterization of hematopoietic stem cells with durable self-renewal potential. Blood. 2009;113(25):6342-6350.

5. Montecino-Rodriguez E, Kong Y, Casero D, et al. Lymphoid-biased hematopoietic stem cells are maintained with age and efficiently generate lymphoid progeny. Stem Cell Reports. 2019;12(3):584-596.

6. Liang Y, Van Zant G, Szilvassy SJ. Effects of aging on the homing and engraftment of murine hematopoietic stem and progenitor cells. Blood. 2005;106(4):1479-1487.
7. Mohrin M, Shin J, Liu Y, et al. Stem cell aging. A mitochondrial UPR-mediated metabolic checkpoint regulates hematopoietic stem cell aging. Science. 2015;347(6228): 1374-1377.

8. Rossi DJ, Bryder D, Zahn JM, et al. Cell intrinsic alterations underlie hematopoietic stem cell aging. Proc Natl Acad Sci U S A. 2005;102(26):9194-9199.

9. Sudo K, Ema H, Morita Y, Nakauchi H. Ageassociated characteristics of murine hematopoietic stem cells. J Exp Med. 2000;192(9):1273-1280.

10. Dykstra B, Olthof S, Schreuder J, Ritsema $M$, de Haan G. Clonal analysis reveals multiple functional defects of aged murine hematopoietic stem cells. J Exp Med. 2011;208(13):2691-2703.

11. Chambers SM, Shaw CA, Gatza C, et al. Aging hematopoietic stem cells decline in function and exhibit epigenetic dysregulation. PLoS Biol. 2007;5(8):e201.

12. Flach J, Bakker ST, Mohrin $M$, et al. Replication stress is a potent driver of functional decline in ageing haematopoietic stem cells. Nature. 2014;512(7513):198-202.

13. Rossi DJ, Bryder D, Seita J, et al. Deficiencies in DNA damage repair limit the function of haematopoietic stem cells with age. Nature. 2007;447(7145):725-729.
14. Florian MC, Nattamai KJ, Dorr K, et al. A canonical to non-canonical Wnt signalling switch in haematopoietic stem-cell ageing. Nature. 2013;503(7476):392-396.

15. Ito K, Suda T. Metabolic requirements for the maintenance of self-renewing stem cells. Nat Rev Mol Cell Biol. 2014;15(4):243-256.

16. Chandel NS, Jasper H, Ho TT, Passegue E. Metabolic regulation of stem cell function in tissue homeostasis and organismal ageing. Nat Cell Biol. 2016;18(8):823-832.

17. Ho TT, Warr MR, Adelman ER, et al Autophagy maintains the metabolism and function of young and old stem cells. Nature. 2017;543(7644):205-210.

18. Vilchez D, Simic MS, Dillin A. Proteostasis and aging of stem cells. Trends Cell Biol. 2014;24(3):161-170.

19. Ergen AV, Boles NC, Goodell MA Rantes/Ccl5 influences hematopoietic stem cell subtypes and causes myeloid skewing. Blood. 2012;119(11):2500-2509.

20. Taichman RS, Emerson SG. Human osteoblasts support hematopoiesis through the production of granulocyte colony-stimulating factor. J Exp Med. 1994;179(5):16771682 .

21. Zhang J, Niu C, Ye L, et al. Identification of the haematopoietic stem cell niche and control of the niche size. Nature. 2003;425 
(6960):836-841

22. Zhao M, Tao F, Venkatraman A, et al. N-cadherin-expressing bone and marrow stromal progenitor cells maintain reserve hematopoietic stem cells. Cell Rep. 2019;26 (3):652-669.

23. Calvi LM, Adams GB, Weibrecht KW, et al. Osteoblastic cells regulate the haematopoietic stem cell niche. Nature. 2003;425 (6960):841-846.

24. Arai F, Hirao A, Ohmura M, et al. Tie2/angiopoietin-1 signaling regulates hematopoietic stem cell quiescence in the bone marrow niche. Cell. 2004;118(2):149161

25. Yoshihara H, Arai F, Hosokawa K, et al. Thrombopoietin/MPL signaling regulates hematopoietic stem cell quiescence and interaction with the osteoblastic niche. Cell Stem Cell. 2007;1(6):685-697.

26. Adams GB, Chabner KT, Alley IR, et al. Stem cell engraftment at the endosteal niche is specified by the calcium-sensing receptor. Nature. 2006;439(7076):599-603.

27. Sugiyama T, Kohara H, Noda M, Nagasawa $\mathrm{T}$. Maintenance of the hematopoietic stem cell pool by CXCL12-CXCR4 chemokine signaling in bone marrow stromal cell niches. Immunity. 2006;25(6):977-988.

28. Kinashi T, Springer TA. Steel factor and c-kit regulate cell-matrix adhesion. Blood. 1994;83(4):1033-1038.

29. Ding L, Morrison SJ. Haematopoietic stem cells and early lymphoid progenitors occupy distinct bone marrow niches. Nature. 2013;495(7440):231-235

30. Greenbaum AM, Revollo LD, Woloszynek JR, Civitelli R, Link DC. N-cadherin in osteolineage cells is not required for maintenance of hematopoietic stem cells. Blood. 2012;120(2):295-302

31. Boulais PE, Frenette PS. Making sense of hematopoietic stem cell niches. Blood. 2015;125(17):2621-2629.

32. Bruns I, Lucas D, Pinho S, et al. Megakaryocytes regulate hematopoietic stem cell quiescence through CXCL4 secretion. Nat Med. 2014;20(11):1315-1320.

33. Zhao M, Perry JM, Marshall $\mathrm{H}$, et al. Megakaryocytes maintain homeostatic quiescence and promote post-injury regeneration of hematopoietic stem cells. Nat Med. 2014;20(11):1321-1326.

34. Nakamura-Ishizu A, Takubo K, Fujioka M, Suda T. Megakaryocytes are essential for HSC quiescence through the production of thrombopoietin. Biochem Biophys Res Commun. 2014:454(2):353-357.

35. Pinho S, Marchand T, Yang E, et al. Lineagebiased hematopoietic stem cells are regulated by distinct niches. Dev Cell. 2018;44(5): 634-641.

36. Florian MC, Dorr K, Niebel A, et al. Cdc42 activity regulates hematopoietic stem cell aging and rejuvenation. Cell Stem Cell. 2012;10(5):520-530

37. Xing Z, Ryan MA, Daria D, et al. Increased hematopoietic stem cell mobilization in aged mice. Blood. 2006;108(7):2190-2197

38. Maryanovich $\mathrm{M}$, Zahalka AH, Pierce $\mathrm{H}$, et al. Adrenergic nerve degeneration in bone marrow drives aging of the hematopoietic stem cell niche. Nat Med. 2018;24(6):782791.

39. Ho YH, Del Toro R, Rivera-Torres J, et al. Remodeling of bone marrow hematopoietic stem cell niches promotes myeloid cell expansion during premature or physiological aging. Cell Stem Cell. 2019;25(3):407418.

40. Sacma M, Pospiech J, Bogeska R, et al.
Haematopoietic stem cells in perisinusoida niches are protected from ageing. Nat Cell Biol. 2019;21(11):1309-1320.

41. Stolzing A, Jones E, McGonagle D, Scutt A. Age-related changes in human bone mar row-derived mesenchymal stem cells: consequences for cell therapies. Mech Ageing Dev. 2008;129(3):163-173.

42. Garcia-Prat L, Sousa-Victor P, MunozCanoves P. Functional dysregulation of stem cells during aging: a focus on skeletal muscle stem cells. FEBS J. 2013;280(17):4051-4062.

43. Siegel G, Kluba T, Hermanutz-Klein U, et al. Phenotype, donor age and gender affect function of human bone marrow-derived mesenchymal stromal cells. BMC Med. 2013;11:146.

44. Wagner W, Bork S, Horn P, et al. Aging and replicative senescence have related effects on human stem and progenitor cells. PloS One. 2009;4(6):e5846

45. Ganguly P, El-Jawhari JJ, Burska AN, et al. The analysis of in vivo aging in human bone marrow mesenchymal stromal cells using colony-forming unit-fibroblast assay and the CD45(low)CD271(+) Phenotype. Stem Cells Int. 2019;2019: 5197983.

46. Visnjic D, Kalajzic Z, Rowe DW, et al. Hematopoiesis is severely altered in mice with an induced osteoblast deficiency. Blood. 2004;103(9):3258-3264.

47. Zhu J, Garrett R, Jung Y, et al. Osteoblasts support B-lymphocyte commitment and differentiation from hematopoietic stem cells. Blood. 2007;109(9):3706-3712.

48. Wu JY, Purton LE, Rodda SJ, et al. Osteoblastic regulation of $\mathrm{B}$ lymphopoiesis is mediated by Gs\{alpha\}-dependent signaling pathways. Proc Natl Acad Sci U S A 2008;105(44):16976-16981.

49. Zehentmeier S, Pereira JP. Cell circuits and niches controlling B cell development. Immunol Rev. 2019;289(1):142-157.

50. Fistonich C, Zehentmeier S, Bednarski JJ, et al. Cell circuits between $B$ cell progenitor and IL-7(+) mesenchymal progenitor cells control B cell development. J Exp Med. 2018;215(10):2586-2599.

51. Cordeiro Gomes A, Hara T, Lim VY, et al. Hematopoietic stem cell niches produce lineage-instructive signals to control multipotent progenitor differentiation. Immunity. 2016:45(6):1219-1231.

52. Balzano M, De Grandis M, Vu Manh TP, et al. Nidogen-1 contributes to the interaction network involved in pro-B cell retention in the peri-sinusoidal hematopoietic stem cell niche. Cell Rep. 2019;26(12):3257-3271

53. Nakahara F, Borger DK, Wei O, et al. Engineering a haematopoietic stem cell niche by revitalizing mesenchymal stromal cells. Nat Cell Biol. 2019;21(5):560-567.

54. Guidi N, Sacma M, Standker L, et al. Osteopontin attenuates aging-associated phenotypes of hematopoietic stem cells. EMBO J. 2017;36(7):840-853.

55. Nilsson SK, Johnston HM, Whitty GA, et al. Osteopontin, a key component of the hematopoietic stem cell niche and regulator of primitive hematopoietic progenitor cells. Blood. 2005;106(4):1232-1239.

56. Stier S, Ko Y, Forkert R, et al. Osteopontin is a hematopoietic stem cell niche component that negatively regulates stem cell pool size. J Exp Med. 2005;201(11):1781-1791.

57. Haylock DN, Nilsson SK. Osteopontin: bridge between bone and blood. $\mathrm{Br}$ Haematol. 2006;134(5):467-474

58. Wintges K, Beil FT, Albers J, et al. Impaired bone formation and increased osteoclastogenesis in mice lacking chemokine (C-C motif) ligand 5 (Ccl5). J Bone Miner Res. 2013;28(10):2070-2080

59. Kim M, Kim C, Choi YS, et al. Age-related alterations in mesenchymal stem cells related to shift in differentiation from osteogenic to adipogenic potential: implication to ageassociated bone diseases and defects. Mech Ageing Dev. 2012;133(5):215-225

60. Zhou BO, Yu H, Yue R, et al. Bone marrow adipocytes promote the regeneration of stem cells and haematopoiesis by secreting SCF. Nat Cell Biol. 2017;19(8):891-903

61. Mancuso P, Bouchard B. The impact of aging on adipose function and adipokine synthesis. Front Endocrinol (Lausanne). 2019; 10:137

62. Ambrosi TH, Scialdone A, Graja A, et al Adipocyte accumulation in the bone marrow during obesity and aging impairs stem cell-based hematopoietic and bone regeneration. Cell Stem Cell. 2017;20(6) 771-784.

63. Fazeli PK, Horowitz MC, MacDougald OA et al. Marrow fat and bone--new perspec tives. J Clin Endocrinol Metab. 2013;98 (3):935-945

64. Schwartz AV. Marrow fat and bone: review of clinical findings. Front Endocrinol (Lausanne). 2015;6:40.

65. Zhang DY, Wang HJ, Tan YZ. Wnt/betacatenin signaling induces the aging of mesenchymal stem cells through the DNA damage response and the $\mathrm{p} 53 / \mathrm{p} 21$ pathway. PloS One. 2011;6(6):e21397.

66. Zheng Y, He L, Wan Y, Song J. H3K9meenhanced DNA hypermethylation of the p16INK4a gene: an epigenetic signature for spontaneous transformation of rat mes enchymal stem cells. Stem Cells Dev. 2013;22(2):256-267.

67. Kornicka K, Marycz K, Tomaszewski KA Maredziak M, Smieszek A. The effect of age on osteogenic and adipogenic differentiation potential of human adipose derived stroma stem cells (hASCs) and the impact of stress fctors in the course of the differentiation process. Oxid Med Cell Longev. 2015;2015: 309169

68. Ju Z, Jiang H, Jaworski M, et al. Telomere dysfunction induces environmental alterations limiting hematopoietic stem cell function and engraftment. Nat Med. 2007;13 (6):742-747

69. Hennrich ML, Romanov N, Horn P, et al Cell-specific proteome analyses of human bone marrow reveal molecular features of age-dependent functional decline. Nat Commun. 2018;9(1):4004.

70. Kusumbe AP, Ramasamy SK, Itkin T, et al Age-dependent modulation of vascular nich es for haematopoietic stem cells. Nature. 2016;532(7599):380-384

71. Kusumbe AP, Ramasamy SK, Adams RH. Coupling of angiogenesis and osteogenesis by a specific vessel subtype in bone. Nature. 2014;507(7492):323-328

72. Poulos MG, Ramalingam P, Gutkin MC, et al. Endothelial transplantation rejuvenates aged hematopoietic stem cell function. J Clin Invest. 2017;127(11):4163-4178.

73. Tikhonova AN, Dolgalev I, $\mathrm{Hu} \mathrm{H}$, et al. The bone marrow microenvironment at singlecell resolution. Nature. 2019;569(7755):222 228.

74. Kovtonyuk LV, Fritsch K, Feng X, Manz MG Takizawa $\mathrm{H}$. Inflamm-aging of hematopoiesis, hematopoietic stem cells, and the bone marrow microenvironment Front Immunol. 2016;7:502

75. Arranz L, Sanchez-Aguilera A, Martin-Perez $\mathrm{D}$, et al. Neuropathy of haematopoietic 
stem cell niche is essential for myeloproliferative neoplasms. Nature. 2014;512(7512):7881.

76. Reynaud D, Pietras E, Barry-Holson K, et al. IL-6 controls leukemic multipotent progenitor cell fate and contributes to chronic myelogenous leukemia development. Cancer Cell. 2011;20(5):661-673.

77. Pietras EM, Mirantes-Barbeito C, Fong S, et al. Chronic interleukin-1 exposure drives haematopoietic stem cells towards precocious myeloid differentiation at the expense of self-renewal. Nat Cell Biol. 2016;18(6): 607-618.

78. Beaulieu LM, Lin E, Mick E, et al. Interleukin 1 receptor 1 and interleukin 1 beta regulate megakaryocyte maturation, platelet activation, and transcript profile during inflammation in mice and humans. Arterioscler Thromb Vasc Biol. 2014;34(3):552-564.

79. Nishimura S, Nagasaki M, Kunishima S, et al. IL-1alpha induces thrombopoiesis through megakaryocyte rupture in response to acute platelet needs. J Cell Biol. 2015;209(3):453-466.

80. Grover A, Sanjuan-Pla A, Thongjuea S, et al. Single-cell RNA sequencing reveals molecular and functional platelet bias of aged haematopoietic stem cells. Nat Commun. 2016;7:11075.

81. Frisch BJ, Hoffman CM, Latchney SE, et al. Aged marrow macrophages expand plateletbiased hematopoietic stem cells via interleukin1B. JCI Insight. 2019;5.pii:124213.

82. Kaser A, Brandacher G, Steurer W, et al. Interleukin-6 stimulates thrombopoiesis through thrombopoietin: role in inflammatory thrombocytosis. Blood. 2001;98(9): 2720-2725.

83. Yamashita M, Passegue E. TNF-alpha Coordinates hematopoietic stem cell survival and myeloid regeneration. Cell Stem Cell. 2019;25(3):357-372.

84. Sakamaki S, Hirayama Y, Matsunaga T, et al. Transforming growth factor-beta1 (TGFbeta1) induces thrombopoietin from bone marrow stromal cells, which stimulates the expression of TGF-beta receptor on megakaryocytes and, in turn, renders them susceptible to suppression by TGF-beta itself with high specificity. Blood. 1999;94(6):1961-1970.

85. Haas S, Hansson J, Klimmeck D, et al. Inflammation-induced emergency megakaryopoiesis driven by hematopoietic stem cell-like megakaryocyte progenitors. Cell Stem Cell. 2015;17(4):422-434.

86. Beerman I, Bhattacharya D, Zandi S, et al. Functionally distinct hematopoietic stem cells modulate hematopoietic lineage potential during aging by a mechanism of clonal expansion. Proc Natl Acad Sci U S A. 2010;107(12):5465-5470.

87. Mann M, Mehta A de Boer CG, et al. Heterogeneous responses of hematopoietic stem cells to inflammatory stimuli are altered with age. Cell Rep. 2018;25(11): 2992-3005.

88. Hernandez G, Mills TS, Rabe JL, et al. Proinflammatory cytokine blockade attenuates myeloid expansion in a murine model of rheumatoid arthritis. Haematologica. 2019 May 17. [Epub ahead of print]

89. Pietras EM. Inflammation: a key regulator of hematopoietic stem cell fate in health and disease. Blood. 2017;130(15):1693-1698.

90. Mendez-Ferrer S, Battista M, Frenette PS Cooperation of beta(2)- and beta(3)-adrenergic receptors in hematopoietic progenitor cell mobilization. Ann N Y Acad Sci. 2010;1192:139-144

91. Katayama Y, Battista M, Kao WM, et al. Signals from the sympathetic nervous system regulate hematopoietic stem cell egress from bone marrow. Cell. 2006;124(2):407421.

92. Mendez-Ferrer S, Lucas D, Battista M Frenette PS. Haematopoietic stem cell release is regulated by circadian oscillations. Nature. 2008:452(7186):442-447.

93. Chartier SR, Mitchell SAT, Majuta LA, Mantyh PW. The changing sensory and sympathetic innervation of the young, adult and aging mouse femur. Neuroscience. 2018;387:178-190.

94. Hart EC, Charkoudian N. Sympathetic neural regulation of blood pressure: influences of sex and aging. Physiology (Bethesda) 2014;29(1):8-15.

95. Ng AV, Callister R, Johnson DG, Seals DR. Age and gender influence muscle sympathetic nerve activity at rest in healthy humans. Hypertension. 1993;21(4):498-503.

96. Veith RC, Featherstone JA, Linares OA, Halter JB. Age differences in plasma norepinephrine kinetics in humans. J Gerontol. 1986;41(3):319-324

97. Ziegler MG, Lake CR, Kopin IJ. Plasma noradrenaline increases with age. Nature. 1976;261(5558):333-335.

98. Chen S, Du C, Shen M, et al. Sympathetic stimulation facilitates thrombopoiesis by promoting megakaryocyte adhesion, migration, and proplatelet formation. Blood. 2016;127(8):1024-1035

99. Pongratz G, Straub RH. The sympathetic nervous response in inflammation. Arthritis Res Ther. 2014;16(6):504

100. Drexler B, Passweg JR, Tzankov A, et al. The sympathomimetic agonist mirabegron did not lower JAK2-V617F allele burden, but restored nestin-positive cells and reduced reticulin fibrosis in patients with myeloproliferative neoplasms: results of phase II study SAKK 33/14. Haematologica. 2019;104(4):710-716.

101. Triposkiadis F, Karayannis G, Giamouzis G, et al. The sympathetic nervous system in heart failure physiology, pathophysiology, and clinical implications. J Am Coll Cardiol. 2009;54(19):1747-1762.

102. Cosentino M, Marino F, Maestroni GJ. Sympathoadrenergic modulation of hematopoiesis: a review of available evidence and of therapeutic perspectives. Front Cell Neurosci. 2015;9:302.

103. Casanova-Acebes M, Pitaval C, Weiss LA, et al. Rhythmic modulation of the hematopoietic niche through neutrophil clearance. Cell. 2013;153(5):1025-1035.

104. De Sandre-Giovannoli A, Bernard R, Cau P, et al. Lamin a truncation in Hutchinson-
Gilford progeria. Science. 2003;300(5628): 2055

105. Eriksson M, Brown WT, Gordon LB, et al. Recurrent de novo point mutations in lamin A cause Hutchinson-Gilford progeria syndrome. Nature. 2003;423(6937):293-298.

106. Merideth MA, Gordon LB, Clauss S, et al. Phenotype and course of HutchinsonGilford progeria syndrome. N Engl J Med. 2008;358(6):592-604

107. Scaffidi P, Misteli T. Lamin A-dependent nuclear defects in human aging. Science. 2006;312(5776):1059-1063.

108. Grigoryan A, Guidi N, Senger K, et al LaminA/C regulates epigenetic and chromatin architecture changes upon aging of hematopoietic stem cells. Genome Biol. 2018;19(1):189.

109. Heo HR, Chen L, An B, et al. Hormonal regulation of hematopoietic stem cells and their niche: a focus on estrogen. Int J Stem Cells 2015;8(1):18-23.

110. Stewart MH, Gutierrez-Martinez P, Beerman I, et al. Growth hormone receptor signaling is dispensable for HSC function and aging. Blood. 2014;124(20):3076-3080.

111. Sanchez-Aguilera A, Arranz L, Martin-Perez $\mathrm{D}$, et al. Estrogen signaling selectively induces apoptosis of hematopoietic progenitors and myeloid neoplasms without harming steady-state hematopoiesis. Cell Stem Cell. 2014;15(6):791-804.

112. Nakada D, Oguro H, Levi BP, et al Oestrogen increases haematopoietic stemcell self-renewal in females and during pregnancy. Nature. 2014;505(7484):555-558.

113. Osorio FG, Navarro CL, Cadinanos J, et al Splicing-directed therapy in a new mouse model of human accelerated aging. Sci Transl Med. 2011;3(106):106ra107.

114. Young K, Borikar S, Bell R, et al. Progressive alterations in multipotent hematopoietic progenitors underlie lymphoid cell loss in aging. J Exp Med. 2016;213(11):2259-2267.

115. Kajimura D, Hinoi E, Ferron M, et al Genetic determination of the cellular basis of the sympathetic regulation of bone mass accrual. J Exp Med. 2011;208(4):841-851.

116. Raaijmakers M. Aging of the hematopoietic stem cell niche: an unnerving matter. Cell Stem Cell. 2019;25(3):301-303.

117. Hoffmann C, Leitz MR, Oberdorf-Maass S, Lohse MJ, Klotz KN. Comparative pharmacology of human beta-adrenergic receptor subtypes--characterization of stably transfected receptors in $\mathrm{CHO}$ cells. Naunyn Schmiedebergs Arch Pharmacol. 2004;369 (2):151-159

118. Deininger MWN, Tyner JW, Solary E. Turning the tide in myelodysplastic/myeloproliferative neoplasms. Nat Rev Cancer. 2017;17(7):425-440

119. Li AJ, Calvi LM. The microenvironment in myelodysplastic syndromes: niche-mediated disease initiation and progression. Exp Hematol. 2017;55:3-18.

120. Wang C, Yang Y, Gao S, et al. Immune dysregulation in myelodysplastic syndrome clinical features, pathogenesis and therapeutic strategies. Crit Rev Oncol Hematol. 2018;122:123-132. 